\title{
Social functioning in children with brain insult
}

\author{
Mardee Greenham ${ }^{1 *}$, Megan M. Spencer-Smith ${ }^{1,2}$, Peter J. Anderson ${ }^{1,2}$, Lee Coleman ${ }^{3}$ and Vicki A. Anderson ${ }^{1,2,3}$ \\ ${ }^{1}$ Murdoch Childrens Research Institute, Melbourne, VIC, Australia \\ 2 University of Melbourne, Melbourne, VIC, Australia \\ ${ }^{3}$ Royal Children's Hospital, Melbourne, VIC, Australia
}

\section{Edited by:}

Silvia A. Bunge, University of California Berkeley, USA

\section{Reviewed by:}

Paul Eslinger, Penn State University, USA

Steve Anderson, University of lowa, USA

Silvia A. Bunge, University of California Berkeley, USA

\section{*Correspondence:}

Mardee Greenham, Critical Care and Neurosciences, Murdoch Childrens Research Institute, Flemington Road, Parkville, VIC 3052, Australia. email:mardee.greenham@mcri.edu.au
Social dysfunction is commonly reported by survivors of brain insult, and is often rated as the most debilitating of all sequelae, impacting on many areas of daily life, as well as overall quality of life. Within the early brain insult (EBI) literature, physical and cognitive domains have been of primary interest and social skills have received scant attention. As a result it remains unclear how common these problems are, and whether factors predictive of recovery (insult severity, lesion location, age at insult, environment) in other functional domains (motor, speech, cognition) also contribute to social outcome. This study compared social outcomes for children sustaining EBI at different times from gestation to late childhood to determine whether EBI was associated with an increased risk of problems. Children with focal brain insults were categorized according to timing of brain insult: (i) Congenital ( $n=38)$ : EBI: first-second trimester; (ii) Perinatal ( $n=33)$; EBI: third trimester to 1-month post-natal; (iii) Infancy $(n=23)$ : EBI: 2 months-2 years post-birth; (iv) Preschool ( $n=19$ ): EBI: 3-6 years; (v) Middle Childhood ( $n=31$ ): EBI: 7-9 years; and (vi) Late Childhood ( $n=19$ ): EBI: after age 10. Children's teachers completed questionnaires measuring social function (Strengths and Difficulties Questionnaire, Walker-McConnell Scale of Social Competence and School Adjustment). Results showed that children with EBI were at increased risk for social impairment compared to normative expectations. EBI before age 2 years was associated with most significant social impairment, while children with EBI in the preschool years and in late childhood recorded scores closer to normal. Lesion location and laterality were not predictive of social outcome, and nor was social risk. In contrast, presence of disability (seizures) and family function were shown to contribute to aspects of social function.

Keywords: social skills, childhood, infant, brain insult, plasticity, vulnerability

\section{INTRODUCTION}

Children, and indeed all humans, live in a world in which they are in constant interaction with other people. These social interactions are based on understanding and storing knowledge about other persons, and about the self, as well as possessing knowledge about interpersonal norms and scripts required to navigate the social world (Adolphs, 2009; Van Overwalle, 2009). Recent advances in the social neurosciences illustrate the close association between these social skills, and underlying brain function (Adolphs, 2009). Further, the development of this knowledge is critical for normal social development, and disruptions to these processes has negative implications for a range of domains including mental health, school progress, and child and family quality of life (Yeates et al., 2007).

Social functioning, and a child's ability to interact appropriately with peers, is an important goal of childhood. Social skills play an integral role in development, and emerge gradually through infancy and childhood. In the first few months of life, the child begins to smile and engage with others, and imitate the actions of those around them in an interactive manner. By $5-8$ months, infants display evidence of goal-directed social behavior (Woodward, 1998). At 3-4 years children can describe the mental states or beliefs of others, distinct from their own (Saxe et al., 2004), and begin to be able to suppress inappropriate responses (Diamond and Taylor, 1996) and participate in pretend play (Goncu et al., 2002). Through this period the child's interest in social relationships emerges with more and more time spent in peer interactions (Howes and Phillipsen, 1998). By 7-8 years children begin to predict the behavior of others based on past experiences (Rholes et al., 1990), and are able to understand and employ non-literal language, such as sarcasm and irony (Demorest et al., 1984). Social decision making and judgments emerge later, in early adolescence (Dodge et al., 2002), and adolescents spend almost a third of their waking hours in social interactions with their peers (Csikszentmihaly and Larson, 1984). During this protracted developmental process, any disruption that impacts on normal maturation processes will have the capacity to impair future progress.

It is likely that social function will be dependent on many factors - both environmental and biological. The influences of family and environmental factors for social development are well established (Ladd and Pettit, 2002). Findings emerging from the social neurosciences suggest that brain-related factors also need to be considered. An example of such a 'disruption' in childhood is brain insult which can result in serious and permanent consequences, in areas such as cognition and academic performance (Ewing-Cobbs et al., 1997; Taylor et al., 2002; Anderson et al., 2005, 2009). Social problems have also been identified (Janusz et al., 2002; Yeates et al., 2004; Ganesalingam et al., 2006) including poor selfesteem, reduced adaptive behaviors, loneliness (Andrews et al. 1998; Yeates et al., 2004), and difficulties in peer relationships (Bohnert et al., 1997). 
While there is some evidence that insult severity may be related to degree of social problems (Andrews et al., 1998; Yeates et al., 2004), this dose-response relationship does not appear to be as clear cut for social skills as it is for physical and cognitive function, where it is well established that more severe insult leads to greater deficits (Anderson et al., 2005). Similarly, there has been little research to date to investigate whether other established predictors of physical and cognitive outcomes following childhood brain insult (for example, location of lesion, age at insult, disability and environmental factors) make a significant contribution to social outcomes after childhood brain insult. In the adult literature, location of lesion has been shown to contribute to social outcome, with the frontal lobes and the right hemisphere designated as playing key roles. These relationships have been less well evaluated in the child-based literature. Anderson et al. (2000) and Eslinger and Biddle (2008) have reported on serial evaluations of two young adult patients who sustained right-sided prefrontal cortex damage prior to 16 months of age. Despite largely intact cognitive abilities, both cases displayed severe and increasing social problems through childhood, including few friendships, rejection by peers, and lack of guilt, empathy, remorse or fear. Spencer-Smith and Anderson (2009) have also examined a small sample of children with subcortical band heterotopia, a congenital disorder of the CNS occurring during gestation and impacting the brain quite globally. They reported that half of their sample were rated by their caregiver as demonstrating impaired social skills, suggesting that, either frontal connections may be disrupted in this condition, or that total brain integrity is important for intact social function.

The objective of this study was to address the gaps in the literature and investigate social functioning in a sample of children with brain insult. We focused on children for whom recovery was expected to be largely complete ( $>12$-months post-insult), and for whom social skill should be well established (10-16 years of age). Our primary aims were to examine the incidence of social difficulties in the context of EBI, and to determine the role of age at insult for outcome. Our secondary aim was to explore factors which might contribute to the emergence of social problems after EBI. Based on the available literature we predicted that: (1) children with EBI would have poorer than expected social functioning; (2) Earlier age at brain insult would be associated with poorer social functioning; (3) lesion characteristics, including location, laterality, presence of seizures would predict social function. Specifically, based on adult literature, we expected poorer social outcome after EBI would be associated with both focal frontal and right hemisphere lesions and presence of seizures; and (4) environmental factors, including higher social risk and poorer family function, would be linked to poor social outcome after EBI.

\section{MATERIALS AND METHODS SAMPLE}

The sample comprised 147 children, including 84 (57.1\%) males, aged between $10-16$ years at recruitment $(M=12.99, \mathrm{SD}=1.88)$, with a history of early brain insult (EBI). This group was part of a larger study looking at a broad range of outcomes from EBI (Anderson et al., 2009). Participants were recruited from the Royal Children's Hospital, Melbourne, Australia between 2005 - 2007. Potential participants were identified via medical record review and referrals to neuroscience outpatient clinics. Inclusion criteria were: (1) 10-16 years at assessment; (2) English speaking; (3) evidence of focal brain insult documented via MRI scan; and (4) brain insult sustained at least 12 months prior to assessment, to allow for stabilization of recovery processes. Approaches were made to 215 families, with 50 families declining to participate (76.7\% participation rate) due to time burden $(n=18)$, lack of interest $(n=29)$, or distance $(n=3)$. Eleven children were excluded from the study based on study criteria. Eighteen cases were excluded due to missing questionnaire data.

Children were then categorized into six 'age at insult' groups, with these chosen based on developmental spurts in neurological and cognitive processes: (1) Congenital $(n=34)$ : EBI during the 1 st and 2 nd trimester of gestation; (2) Perinatal $(n=29)$ : 3 rd trimester of gestation to 1-month post-birth; (3) Infancy $(n=21)$ : 2 months to 2 years; (4) Preschool ( $n=18$ ); 3-6 years; (5) Middle Childhood $(n=30)$ : $7-9$ years; and (6) Late Childhood $(n=15)$ : 10-16 years.

Mechanism of EBI was diverse and included developmental (dysplasia, heterotopia, polymicrogyria), ischemic (periventricular leukomalacia, infarct, stroke), neuroplastic (tumor, cyst, abscess), traumatic (penetrating head injury) and infective (encephalitis, meningitis) (see Table 1).

\section{MATERIALS}

\section{Descriptive information}

Demographic information. Parents completed a questionnaire providing information on their child's medical and developmental history.

Social risk. The Social Risk Index - SRI (Roberts et al., 2008): rates a number of family factors, such as maternal age at the child's birth and primary caregiver's level of education and income. High scores indicate high social risk. Median split was used to determine high social risk and low social risk.

Family functioning. The General Family Functioning scale from the Family Assessment Device (Miller et al., 1985) provided a measure of family functioning. Low scores indicate better family functioning. Median split was used to determine high family functioning and low family functioning.

General intelligence. The four-subtest version of the Wechsler Abbreviated Scale of Intelligence (Wechsler, 1999) was administered. A Full Scale Intelligence Quotient (FSIQ) $(M=100)$, $\mathrm{SD}=15$ ) was derived.

\section{Brain insult characteristics}

MRI scans. i) Acquisition MRI scans were conducted, via standard protocol, as part of routine clinical practice prior to recruitment. For those who had not undergone scanning, or whose scans were unavailable, scans were conducted simultaneously with neurobehavioral evaluation. Scans were conducted on a 1.5 Tesla scanner, and axial and coronal slices were obtained. ii) Coding protocol A coding protocol developed by Leventer et al. (1999) was modified and employed to describe brain insult characteristics including: brain regions affected (lobes, subcortical structures), laterality 
Table 1 | Characteristics for the sample across early brain insult groups.

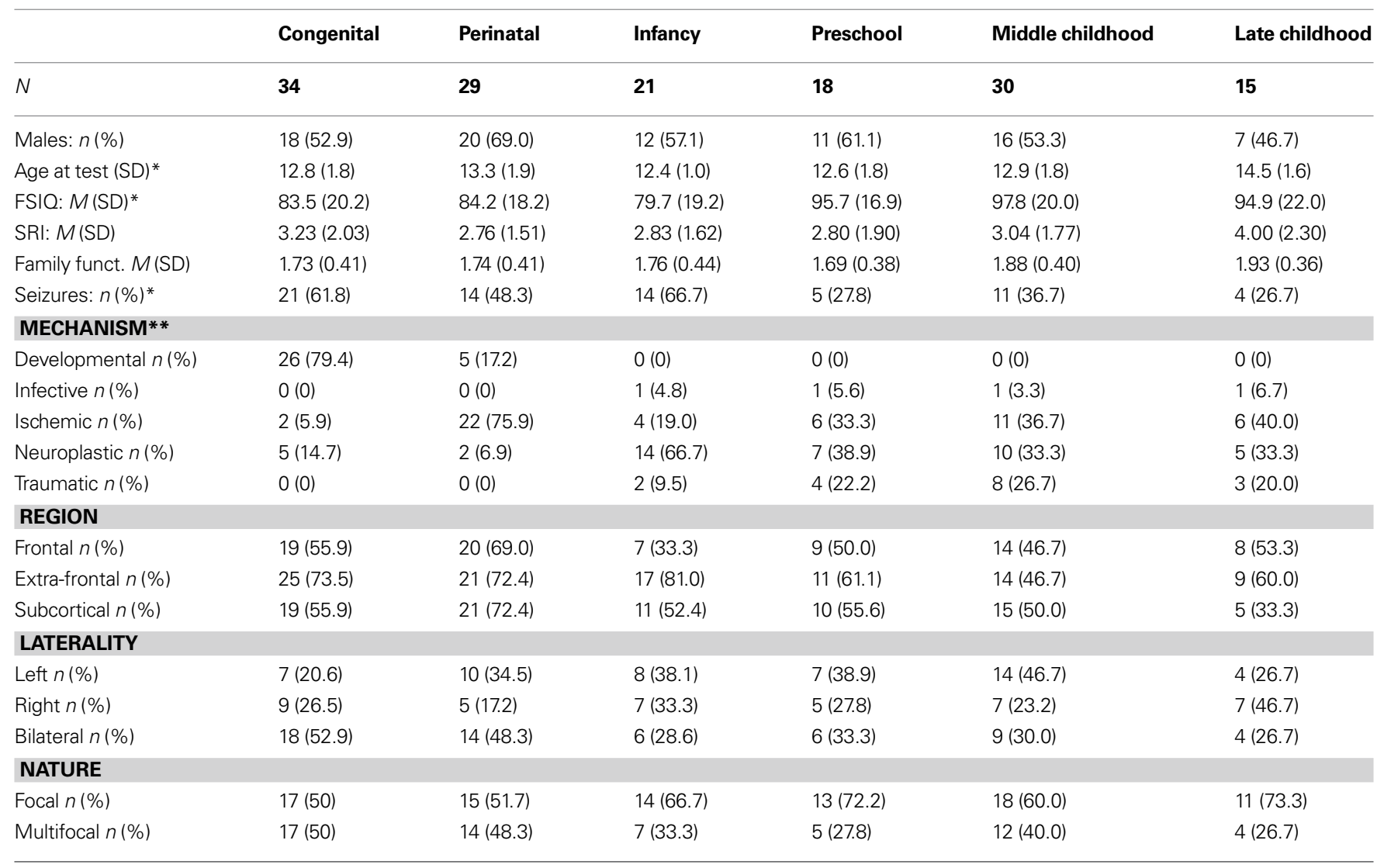

${ }^{*} p<0.05 ;{ }^{*} p<0.001$.

(left, right, bilateral), extent of insult (focal, multifocal), volume of brain affected (number of regions) (Table 2). Scans were coded by an experienced paediatric neuroradiologist (L.C) and neuropsychologist (M.S.S), blind to group membership. A randomly selected subset of 10 scans was re-coded independently by L.C and M.S.S, with inter-rater reliability of 0.97 .

Timing of brain insult was determined via a combination of MRI, brain biopsy, and medical record (clinical history, medical investigations). For pre and perinatal insults this information was reviewed by an experienced paediatric neurologist (R.L) and a neuropsychologist (M.S.S), and rated according to the coding established by Leventer et al. (1999). Ten cases were double-rated, with $100 \%$ consistency. Mechanism of insult was coded as: developmental, infective, ischemic, neuroplastic, or traumatic. Presence of seizure history was recorded.

\section{Outcome measures}

Social functioning was measured by questionnaires completed by the child's teacher, who was asked to rate their student's behavior over the previous 6 months.

The strengths and difficulties questionnaire - SDQ. Goodman (1997) provided a measure of social, behavioral and emotional functioning. While this study specifically addressed social function, all SDQ subscales were included in analyses to provide information on areas of function (behavior, emotions) closely related to social
Table 2 | Definitions for classification of lesion location, laterality and extent, based on MRI scan (adapted from Leventer et al., 1999).

\begin{tabular}{ll}
\hline Variable & Definition \\
\hline REGION & $\begin{array}{l}\text { Brain pathology involves the frontal lobe. } \\
\text { Brontal } \\
\text { Extra-frontal } \\
\text { occipital or temporal lobe only. } \\
\text { Brain pathology involves the corpus callosum, } \\
\text { thalamus or basal ganglia. }\end{array}$ \\
Subcortical & $\begin{array}{l}\text { Brain pathology involves the brain stem } \\
\text { or cerebellum. }\end{array}$ \\
LATERALITY & Brain pathology confined to left hemisphere. \\
Left hemisphere & Brain pathology confined to right hemisphere. \\
Right hemisphere & Brain pathology located in both left and \\
Bilateral & right hemispheres. \\
EXTENT & Brain pathology is confined to one area. \\
Focal & Brain pathology involves two or more areas. \\
Multifocal &
\end{tabular}

function. The questionnaire consists of 25 items, providing five subscales: Emotional Symptoms (ES) (e.g., often unhappy, often seems worried, nervous or clingy in new situations), Conduct 
Problems (CP) (e.g., often has temper tantrums, is disobedient, often lies or cheats), Hyperactivity-Inattention (HYP) (e.g., easily distracted, restless, overactive), Peer Problems (PP) (e.g., tend to play alone, bullied by other children), and Prosocial Behaviors (PB) (e.g., considerate of others, often volunteers to help others). A Total Difficulties (SDQ:TD) score was obtained by combining the scores for all but the PB subscale, consistent with manual instructions. Higher scores on PB indicate better social functioning, while, higher scores on all other subscales and SDQ:TD indicate poorer social functioning. Scaled scores were derived based on published Australian norms (Mellor, 2005) and scores were ranked as Normal, Borderline or Abnormal consistent with manual instructions.

The Walker-McConnell scale of social competence and school adjustment: adolescent version - WMS. Walker and McConnell (1995) was used to identify social skills deficits. It consists of 53 items, from which four subscales are obtained: Self Control (SC) (e.g., reacts to situations without becoming violet or destructive, can accept not getting his/her own way, gains peers' attention in an appropriate manner); Peer Relations (PR) (e.g., making friends, interacting with peers, engaging in conversation with peers); School Adjustment (SA) (e.g., displays independent study skills, listens while others are speaking); and Empathy (EM) (e.g., sensitive to the need of others, compliments others regarding personal attributes). These subscales combine to provide a Total score (WMS:TOT). Scores were converted to scaled scores $(M=100, \mathrm{SD}=15$ for TOT; $M=10, \mathrm{SD}=3$ for subscales) with a lower score indicating poorer social skills.

\section{PROCEDURE}

This study represents a subset of a larger study of children with EBI (Anderson et al., 2009). The study was approved by the Human Research Ethics Committee, Royal Children's Hospital, Melbourne, Australia. Eligible families were initially identified via medical records, neuroradiology meetings or outpatient clinics. Families were mailed details of the study and requests for written consent. Consenting families were first screened and then attended for interview at an outpatient clinic, with a small number of children assessed at home, for family convenience. Parents completed the demographic questionnaire, FAD and SRI in a single session, while their child underwent intellectual evaluation with a trained child psychologist. Following consent from families, the children's teachers were contacted via mail and asked to complete the SDQ and the WMS, based on observations over the previous 6 months. Completed questionnaires were returned directly to researchers in the envelope provided.

\section{STATISTICAL ANALYSIS}

Quantitative analyses were conducted using SPSS (version 17.0).

To address research question 1, that children with EBI would have poorer than expected social functioning, total group mean ratings on subscales of the SDQ and WMS were compared to normative expectations using single sample $t$-tests. Research question 2, that earlier age at brain insult would be associated with poorer social functioning, was examined using one-way ANOVA to identify differences in mean ratings between the six EBI groups, effect size determined by $\eta^{2}$, and post hoc analyses were performed using Turkey's honestly significant differences test. Age at assessment was not included as a covariate because age-scaled scores were examined. Chi Square test-for-Independence analyses investigated differences in rates of impairment between the EBI groups, effect size determined by Cramer's $V$. Research question 3 was examined by using ANCOVA to identify group differences with respect to lesion characteristics (location, laterality, presence of seizures) and environmental factors (social risk, family function). These analyses also included effect size, determined by $\eta^{2}$, and post hoc analyses using Turkey's honestly significant differences test.

\section{RESULTS}

\section{Sample characteristics}

No group differences were identified for gender, social risk, or family functioning (see Table 1). A significant age at test difference was identified, $F(5,146)=3.09, p=0.01, \eta^{2}=0.10$, with the Late Childhood group older at the time of assessment than the Congenital $(p=0.02)$, Infancy $(p=0.007)$, Preschool $(p=0.04)$, and Middle Childhood $(p=0.05)$ groups. General intelligence differed between groups, $F(5,139)=3.67, p=0.004, \eta^{2}=0.21$, with the Middle Childhood group performing better than the Congenital $(p=0.05)$ and Infancy $(p=0.02)$ groups. Significant group differences were also detected for presence of seizures $\chi^{2}(5, n=143)=11.09, p=0.04, V=0.29$, with a larger proportion of children with a history of seizures in the Congenital and Infancy groups and a smaller proportion in the Preschool and Later Childhood groups.

Mechanism of EBI differed between the groups, $\chi^{2}$ (20, $n=146)=130.10, p=<0.001, V=0.47$, consistent with the heterogeneity of the sample (see Table 1). However, there were no statistically significant group differences for: (1) region of insult, frontal, $\chi^{2}(5, n=147)=6.86, p=0.23, V=0.22$, extrafrontal, $\chi^{2}(5, n=147)=8.91, p=0.11, V=0.25$, subcortical, $\chi^{2}$ $(5, n=147)=6.89, p=0.24, V=0.22$; (2) Laterality (left, right, bilateral), $\chi^{2}(5, n=147)=11.59, p=0.31, V=0.20$; or (3) extent of insult (unifocal, multifocal), $\chi^{2}(5, n=147)=4.86, p=0.43$, $V=0.18$, suggesting groups did not differ greatly with respect to these brain insult characteristics.

\section{DO CHILDREN WITH EBI HAVE POORER SOCIAL FUNCTIONING THAN POPULATION DATA WOULD EXPECT?}

The total group of children with EBI performed poorer than normative expectations on most measures of social functioning (see Table 3). For the SDQ, poorer results were identified for most subscales: ES, $t(147)=7.73, p<0.001$; HYP, $t(147)=7.68, p<0.001$; PP, $t(147)=5.43, p<0.001$ PB, $t(147)=-5.43, p<0.001$; SDQ: $\mathrm{TD}, t(147)=8.21, p<0.001$, with the difference between the total group and normative expectations approaching significance on CP, $t(147)=1.87, p=0.06$. For the WMS, there was a significant discrepancy from normative expectations only for $\mathrm{PR}$, $t(144)=-3.17, p<0.001$, suggestive of difficulties with peer relations. The total group performed close to normative expectations on SC, $t(144)=0.36, p=0.72 ; \mathrm{SA}, t(144)=0.28, p=0.78 ; \mathrm{EM}$, $t(144)=1.10, p=0.27$; WMS:TOT, $t(144)=-0.20, p=0.84$.

\section{DOES AGE AT BRAIN INSULT IMPACT ON SOCIAL FUNCTIONING? Differences in social functioning across EBI groups}

On the SDQ, there was a significant group difference for ES, $F(5$, $146)=2.45, p=0.04, \eta^{2}=0.08$, with the Congenital group performing poorer than the Preschool group $(p=0.04)$ (see Table 4). There 
Table 3 | Comparison of EBI groups and normative data for the SDQ andWMS: mean differences and confidence intervals.

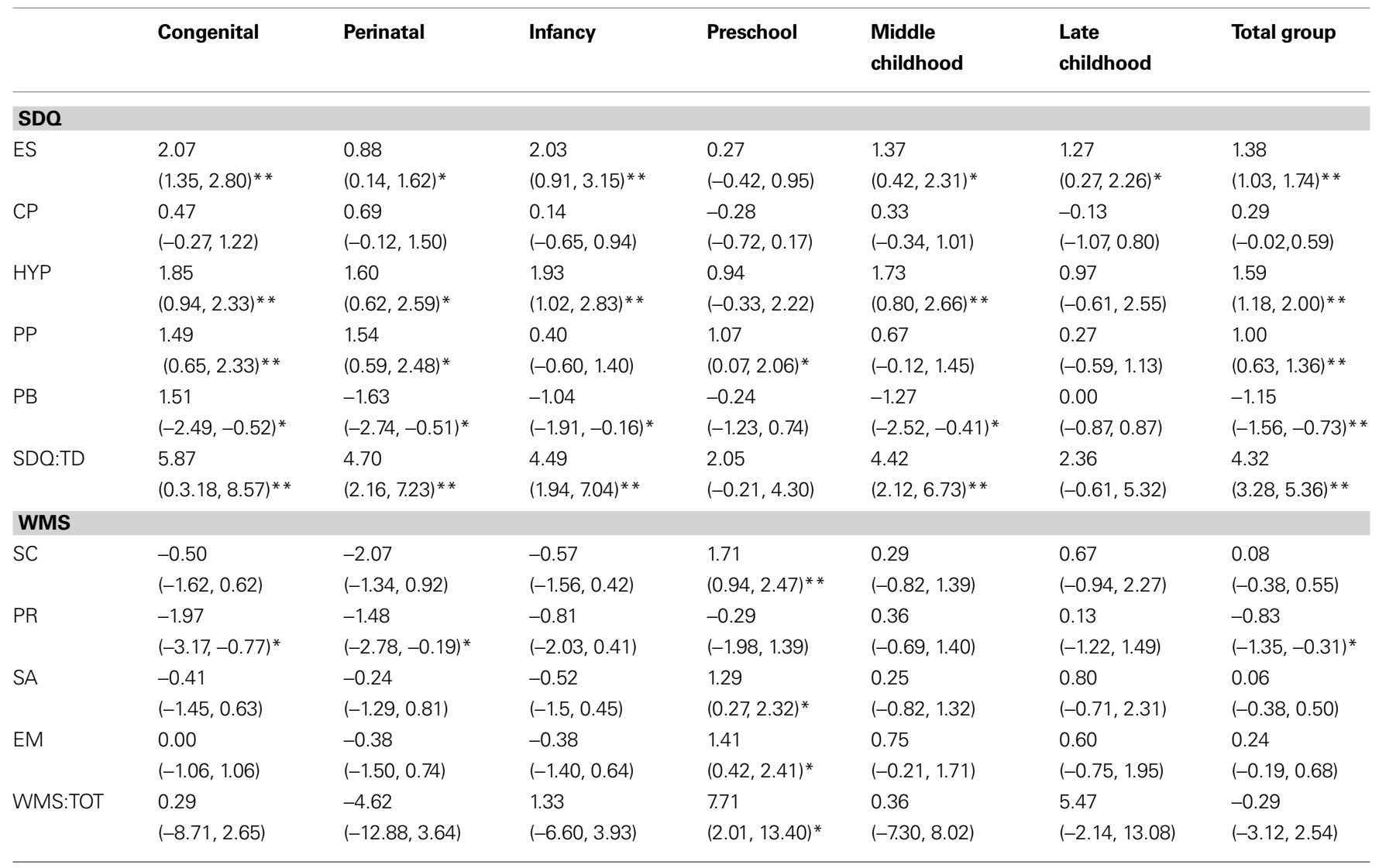

${ }^{*} p<0.05 ;{ }^{*} p<0.001$.

Table 4 | Differences in social functioning ratings across EBI groups.

\begin{tabular}{|c|c|c|c|c|c|c|c|}
\hline & $\begin{array}{l}\text { Congenital } \\
\text { M (SD) }\end{array}$ & $\begin{array}{l}\text { Perinatal } \\
\text { M (SD) }\end{array}$ & $\begin{array}{l}\text { Infancy } \\
\text { M (SD) }\end{array}$ & $\begin{array}{l}\text { Preschool } \\
\text { M (SD) }\end{array}$ & $\begin{array}{l}\text { Middle childhood } \\
\text { M (SD) }\end{array}$ & $\begin{array}{l}\text { Late childhood } \\
\text { M (SD) }\end{array}$ & $\begin{array}{l}\text { Total group } \\
\text { M (SD) }\end{array}$ \\
\hline \multicolumn{8}{|c|}{ SDQ } \\
\hline$E S^{*}$ & $3.47(2.08)$ & 2.28 (1.94) & $3.43(2.46)$ & 1.67 (1.37) & $2.77(2.53)$ & $2.67(1.80)$ & $2.78(0.18)$ \\
\hline $\mathrm{CP}$ & $1.47(2.14)$ & $1.69(2.14)$ & $1.14(1.74)$ & $0.74(0.90)$ & $1.33(1.81)$ & 0.87 (1.69) & $1.29(1.85)$ \\
\hline HYP & $4.35(2.63)$ & $4.10(2.58)$ & 4.43 (1.99) & $3.44(2.57)$ & $4.23(2.49)$ & $3.47(2.85)$ & $4.09(2.51)$ \\
\hline TD & $12.38(7.73)$ & $11.21(6.67)$ & $11.00(5.60)$ & $8.56(4.53)$ & $10.93(6.81)$ & $8.87(5.36)$ & $10.83(6.38)$ \\
\hline \multicolumn{8}{|c|}{ WMS } \\
\hline SC & $9.50(3.20)$ & $9.79(2.70)$ & $9.43(2.18)$ & 11.71 (1.49) & $10.29(2.84)$ & $10.67(2.90)$ & $10.08(2.81)$ \\
\hline$P R^{*}$ & $8.03(3.43)$ & $8.52(3.40)$ & $9.19(2.68)$ & $9.71(3.27)$ & $10.36(2.70)$ & $10.13(2.45)$ & $9.17(3.15)$ \\
\hline SA & $9.59(2.98)$ & $9.76(2.76)$ & $9.48(2.14)$ & 11.29 (1.99) & $10.25(2.76)$ & $10.80(2.73)$ & $10.06(2.67)$ \\
\hline
\end{tabular}

${ }^{*} p<0.05$.

were no significant group differences for the remaining subscales: $\mathrm{CP}$, $F(5,146)=0.85, p=0.51, \eta^{2}=0.03 ; \operatorname{HYP}, F(5,146)=0.59 p=0.71$, $\eta^{2}=0.02 ; \mathrm{PP}, F(5,146)=1.45, p=0.21, \eta^{2}=0.05 ; \mathrm{PB}, F(5,146)=1.51$, $p=0.19, \eta^{2}=0.05$; TD, $F(5,146)=1.18, p=0.32, \eta^{2}=0.04$.
On the WMS, there was a significant group difference for PR, $F(5,143)=2.43, p=0.04, \eta^{2}=0.08$, with the Congenital group performing poorer than the Middle Childhood group $(p=0.04)$ (see Table 3 ). There were no significant group differences for the 
remaining subscales: $\mathrm{SC}, F(5,143)=1.94, p=0.09, \eta^{2}=0.07$; SA, $F(5,143)=1.49, p=0.20, \eta^{2}=0.05 ; \mathrm{EM}, F(5,143)=1.60, p=0.18$, $\eta^{2}=0.05 ;$ TOT, $F(5,143)=1.68, p=0.14, \eta^{2}=0.06$.

\section{Differences in impairment rates across EBI groups}

For the SDQ, the difference in impairment ratings between groups did not reach significance for: $\mathrm{ES}, \chi^{2}(5,147)=7.04, p=0.22$, $V=0.22 ; \mathrm{CP}, \chi^{2}(5,147)=6.95, p=0.22, V=0.22, \mathrm{HYP}, \chi^{2}(5$, $147)=3.38, p=0.64, V=0.15$; PP, $\chi^{2}(5,147)=1.99, p=0.85$, $V=0.12 ; \mathrm{PB}, \chi^{2}(5,147)=7.62, p=0.18, V=0.23 ; \mathrm{TD}, \chi^{2}(5$, $147)=5.29, p=0.38, V=0.19$ (see Figure 1A).

There was a significant difference on the WMS between groups for PR, $\chi^{2}(5,144)=13.74, p=0.02, V=0.31$, with a higher proportion of children impaired in the Congenital groups $(47.1 \%)$ and a smaller proportion of children impaired in the Late Childhood group $(6.7 \%)$. The difference between groups approached significance for SC, $\chi^{2}(5,144)=9.60, p=0.09, V=0.26$, and EM, $\chi^{2}(5$, $144)=9.60, p=0.09, V=0.26$. The Perinatal group had a higher proportion of children impaired on the SC (31\%) and EM (31\%) subscales while the Preschool group had no children with impairments on these subscales. There were no significant differences between groups for: $\mathrm{SA}, \chi^{2}(5,144)=7.96, p=0.16, V=0.24$; or TOT, $\chi^{2}(5,144)=9.68, p=0.09, V=0.26$ (see Figure 1B).

\section{DO LESION CHARACTERISTICS (LOCATION, LATERALITY, PRESENCE OF SEIZURES ) IMPACT ON SOCIAL OUTCOMES FOLLOWING EBI? Lesion location}

On the SDQ and WMS, ANCOVA (covarying for age at insult and seizures) identified no significant differences in outcomes between children with frontal brain pathology and those with lesions to extra-frontal regions only on any subscales (see Table 5). Similarly, no significant differences were identified on the SDQ or WMS for children with EBI confined to the left hemisphere, right hemisphere, or bilateral lesions on any subscales.

\section{History of seizures}

Group differences between children with and without seizure history approached significance on the SDQ for PP, $F(1,142)=0.3 .61$, $\mathrm{p}=0.06, \eta^{2}=0.03$, and children with presence of seizure history had poorer ratings. No differences between groups were found on any other subscales. On the WMS there was a significant difference in outcome between children with and without presence of seizure history on $\mathrm{SC}, F(1,139)=4.17, p=0.04, \eta^{2}=0.03$; PR, $F(1,139)=5.14$, $p=0.03, \eta^{2}=0.04$; and $S A, F(1,139)=5.62, p=0.02, \eta^{2}=0.04$. For these subscales children with presence of seizure history had poorer ratings than children without presence of seizure history. Differences between groups approached significance for EM, $F(1,139)=3.66$, $p=0.06, \eta^{2}=0.03$ and TOT, $F(1,139)=3.60, p=0.06, \eta^{2}=0.03$, suggesting that children with presence of seizure history had poorer ratings than children without presence of seizure history.

\section{DO ENVIRONMENTAL FACTORS (SOCIAL RISK, FAMILY FUNCTION) IMPACT ON SOCIAL OUTCOMES FOLLOWING EBI?}

There were no significant differences between children with high social risk and low social risk on any subscales on the SDQ or WMS (see Table 6). On the SDQ, there was a significant difference between children with low family functioning and children with high family functioning on $\mathrm{ES}, F(1,146)=5.04, p=0.03, \eta^{2}=0.03$, and $\mathrm{PB}, F(1,146)=5.65, p=0.02, \eta^{2}=0.04$, showing that children with low family functioning had poorer outcomes than children with high family functioning. There were no significant differences between groups on the remaining subscales. No significant differences between groups were identified on subscales of the WMS.

\section{DISCUSSION}

The primary purpose of this study was, first, to determine whether children with EBI were at increased risk of social dysfunction, and, if so, to examine whether the impact of age at insult on social function mimics that documented for cognitive skills. Our secondary aim was to explore the impact of lesion characteristics, seizure history and environmental factors on social functioning. Based on adult literature we predicted that, for the biological dimension, frontal pathology, right hemisphere involvement and presence of seizures would be associated with poorer social outcomes. From an environmental perspective, high social risk and family dysfunction were expected to be related to social dysfunction. Our results suggest that, as a group, children with EBI were at increased risk of social dysfunction, with elevated scores across a broad range of measures. EBI before age 3 years conferred particular risk, but significant effects were confined to the domains of peer relations and emotional symptoms. Presence of seizures and family dysfunction also contributed to social outcomes, while lesion location and social risk did not.

\section{DO CHILDREN WITH EBI HAVE POORER SOCIAL FUNCTIONING THAN POPULATION DATA WOULD EXPECT?}

Children with brain insult, as a group, performed poorer than normative expectations on most measures (5/6 subscales on the Strengths and Difficulties Questionnaire, 1/5 on the Walker-McConnell Scale). While mean ratings for the EBI group were not severely impaired (that is, generally falling within 1 SD of expectations), the group were rated to have fewer prosocial behaviors than typically developing peers, with difficulties in peer relationships identified on both the WMS and the SDQ. Children with EBI also experienced significantly more emotional symptoms and hyperactivity than population expectations. Somewhat surprisingly, children with EBI did not demonstrate significant deviations from normal for self-control, school adjustment or empathy. Our findings are in line with previous group-based studies examining children with brain injury (Bohnert et al., 1997; Andrews et al., 1998; Janusz et al., 2002; Ganesalingam et al., 2006; Yeates et al., 2007) and case reports (Anderson et al., 2000; Eslinger and Biddle, 2008; Spencer-Smith and Anderson, 2009) which have consistently identified persisting social and behavioral problems in children with a variety of diagnoses (e.g., traumatic brain injury, stroke, tumor).

\section{DOES DEVELOPMENTAL AGE OF EBI IMPACT ON SOCIAL FUNCTIONING?}

Our results provided limited evidence for the role of age of insult in predicting social difficulties, especially when considering group mean performances. Group differences were identified only for emotional symptoms and peer relations, with the Congenital group recording poorest scores in each case. Examination of impairment ratings yielded additional findings. On the SDQ (Total score) approximately half of the Congenital, Infancy and Middle Childhood 


\section{A $\quad \%$ impaired across EBI groups on the SDQ}
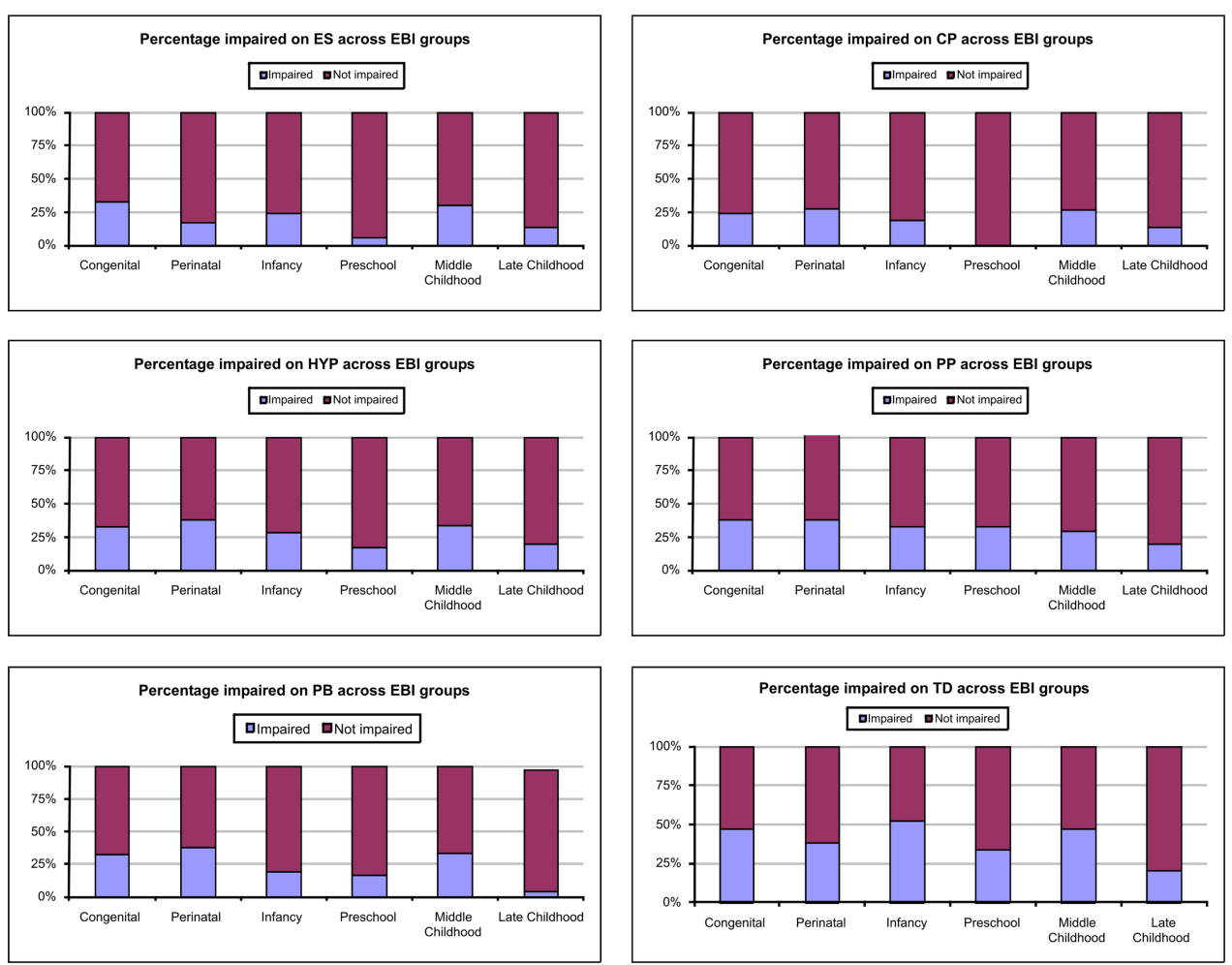

B

\% impaired across EBI groups on the WMS
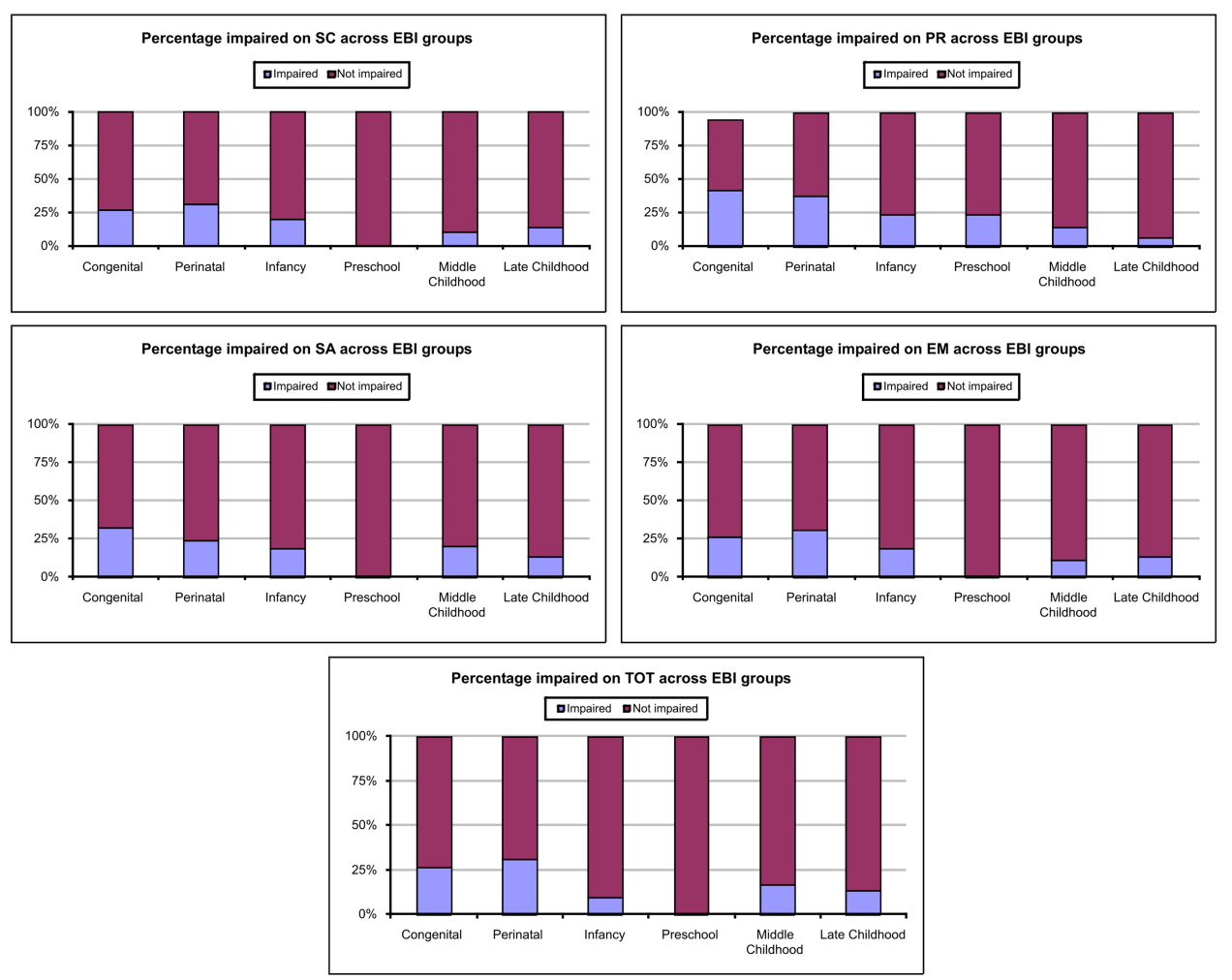

FIGURE 1 | (A) Percentages of children across EBI groups with impairment on the SDQ. (B) Percentage of children across groups identified as impaired on the WCS 
Table 5 | Differences in social functioning ratings for lesion characteristics and seizure history.

\begin{tabular}{|c|c|c|c|c|c|c|c|}
\hline & \multicolumn{2}{|c|}{ Lesion location } & \multicolumn{3}{|c|}{ Lesion laterality } & \multicolumn{2}{|c|}{ Seizures } \\
\hline & Frontal M (SD) & No frontal M (SD) & Left M (SD) & Right M (SD) & Bilateral M (SD) & Yes M (SD) & No M (SD) \\
\hline \multicolumn{8}{|c|}{ SDQ } \\
\hline ES & $2.82(2.16)$ & $2.74(2.20)$ & $2.78(2.03)$ & $2.60(2.02)$ & $2.91(2.40)$ & $2.65(2.04)$ & $2.99(2.34)$ \\
\hline $\mathrm{CP}$ & $1.21(1.85)$ & $1.37(1.87)$ & $1.04(1.64)$ & $1.58(2.02)$ & $1.30(1.91)$ & $1.11(1.59)$ & $1.45(2.01)$ \\
\hline PB & $6.65(2.66)$ & $6.66(2.46)$ & $6.56(2.61)$ & $6.75(2.99)$ & $6.67(2.21)$ & $6.99(2.39)$ & $6.30(2.66)$ \\
\hline TD & $10.62(6.38)$ & $11.06(6.41)$ & $10.22(6.00)$ & $11.55(6.56)$ & $10.86(6.62)$ & $10.01(5.61)$ & $11.72(6.94)$ \\
\hline \multicolumn{8}{|c|}{ WMS } \\
\hline SC & $10.28(2.63)$ & $9.87(2.99)$ & $10.65(2.65)$ & $10.05(2.94)$ & $9.61(2.80)$ & $10.57(2.52)^{*}$ & $9.62(2.98)^{*}$ \\
\hline PR & $9.39(2.89)$ & $8.93(3.42)$ & $9.31(3.13)$ & $9.23(3.10)$ & $9.00(3.26)$ & $9.75(3.16)^{*}$ & $8.54(3.13)^{*}$ \\
\hline
\end{tabular}

${ }^{*} p<0.05$.

Table 6 | Differences in social functioning ratings for environmental factors.

\begin{tabular}{llllll}
\hline & \multicolumn{2}{c}{ Social risk } & & \multicolumn{2}{c}{ Family function } \\
\cline { 6 - 6 } \cline { 6 - 6 } & High M (SD) & Low M (SD) & & Poor M (SD) & Good M (SD) \\
\hline SDO & & & & & \\
ES & $2.75(2.29)$ & $2.86(1.86)$ & & $3.18(2.27)^{*}$ & $2.38(2.00)^{*}$ \\
CP & $1.29(1.83)$ & $1.28(1.93)$ & & $1.45(2.04)$ & $1.12(1.65)$ \\
HYP & $4.10(2.62)$ & $4.07(2.25)$ & & $4.28(2.59)$ & $3.89(2.41)$ \\
PP & $2.54(2.27)$ & $2.74(2.16)$ & & $2.53(2.17)$ & $2.67(2.30)$ \\
PB & $6.56(2.74)$ & $6.88(2.08)$ & & $6.16(2.54)^{*}$ & $7.15(2.50)^{*}$ \\
TD & $10.78(6.59)$ & $10.95(5.90)$ & & $11.43(6.67)$ & $10.22(6.05)$ \\
WMS & & & & & \\
SC & $10.10(2.82)$ & $10.05(2.80)$ & & $10.28(2.83)$ & $9.89(2.78)$ \\
PR & $9.35(3.26)$ & $8.74(2.88)$ & & $9.17(2.90)$ & $9.16(3.40)$ \\
SA & $10.14(2.85)$ & $9.88(2.24)$ & & $9.94(2.63)$ & $10.18(2.73)$ \\
EM & $10.33(2.71)$ & $10.05(2.55)$ & & $10.15(2.59)$ & $10.33(2.74)$ \\
Total & $99.65(18.82)$ & $99.84(12.70)$ & & $100.90(13.63$ & $98.55(20.07)$
\end{tabular}

${ }^{*} p<0.05$.

groups (47.1\%, 52.4\%, 48.7\% respectively), and one-third of the Perinatal and Preschool groups (37.9\%, 33.3\% respectively) fell in the impaired range. While still elevated, impairment was less common in the Late Childhood group (20.0\%). Inspection of the data illustrated in Figure 1A indicates fairly consistent levels of dysfunction across subscales for children sustaining EBI before 3 years of age. In children with later EBI, insults between 7 and 9 years appear to result in similar levels of impairment to those of younger children. Overall, outcome was best for children with insults in the preschool period or late in childhood. Preschool insults were associated with low rates of emotional symptoms and conduct problems, while late childhood insults led to relatively few problems with emotional symptoms, conduct disorder or prosocial behaviors.
For the WMS, impairment rates were lower overall. As for the SDQ, Preschool insults were the most benign, with no child falling in the impaired range in any area with the exception of peer relationships. The Congenital $(26.5 \%)$ and Perinatal (31.0\%) groups recorded highest rates of social impairment overall. In contrast, the Late Childhood, Middle Childhood and Infancy groups recorded low rates of impairment (13.3\%, 16.7\%, 9.5\% respectively) and none of the Preschool group fell in the impaired range.

Our findings indicate that, in contrast to the age-related cognitive deficits documented following EBI (Jacobs et al., 2007; Anderson et al., 2009), social functions appear to be less clearly linked to neurodevelopmental processes. Children sustaining EBI prior to age 3 years do demonstrate a tendency to poorer social and behavioral function, however outcomes appear less linked to age at insult for later insults. Consistent with these results, emerging research has begun to explore behavioral consequences of EBI and likely risk and resilience factors. Findings suggest that, for domains such as social and behavioral function, environmental influences, child disability and associated levels of child adjustment may play a greater role (Janusz et al., 2002; Anderson et al., 2006) than injury-related factors, especially in the long-term post-insult.

\section{DO LESION CHARACTERISTICS, PRESENCE OF SEIZURES OR ENVIRONMENTAL FACTORS IMPACT ON SOCIAL OUTCOMES?}

Research investigating cognitive functioning in children with EBI has established a strong relationship between outcomes and lesion characteristics (e.g., severity, location), seizure history and environmental factors (Anderson et al., 2002, 2005). We observed a somewhat different pattern when examining social function in children with EBI. Findings indicated no significant relationship between lesion characteristics and social function. Specifically, children's social outcomes did not appear to be dependent upon whether lesions involved the frontal lobes or extra-frontal areas. Similarly, laterality of lesion was not predictive of social outcomes or behavioral skills. 
With respect to disability, as has been previously reported for cognition (Hartel et al., 2004; Chilosi et al., 2005; Ballantyne et al., 2007), presence of seizures was found to impact social functioning. Children with seizure history were rated as having more peer problems and more difficulties with school adjustment than children who had not experienced seizures. Lower self-control was also identified.

For environmental factors, social risk, as measured in this study, did not impact social functioning in children with EBI. Social function was not significantly related to high or low social risk (determined by family structure, maternal education, employment status and occupation of primary income earner, language spoken at home and maternal age at birth of the child). In contrast, family functioning was found to impact on the prosocial behavior and emotional subscales of the SDQ, with children from more dysfunctional families at higher risk for impairment in these domains.

\section{LIMITATIONS AND FUTURE DIRECTIONS}

When interpreting study findings, a number of potential limitations should be considered. First, use of normative data rather than an appropriately constructed healthy comparison group is a study limitation. Of importance, our results are consistent with previous research documenting the detrimental effects of brain insult sustained early in life (Ewing-Cobbs et al., 1997; Anderson et al., 2005; Chilosi et al., 2005; Jacobs et al., 2007) and provide little evidence to corroborate early plasticity notions, which argue for good outcome from EBI. Use of normative data does have an advantage over use of small, unrepresentative control samples (e.g., with inflated IQ scores) commonly reported in this field, which increase the risk of inaccurate characterisation of the study results (Ballantyne et al., 2008). Second, this study chose to recruit children based on developmental age at insult rather than the traditional 'condition-based' approach. In doing so, the sample necessarily included children for whom mechanism of insult varied, creating the risk that findings

\section{REFERENCES}

Adolphs, R. (2009). The social brain: neural basis for social knowledge. Ann. Rev. Psychol. 60, 693-716.

Anderson, S. W., Damasio, H., Tranel, D., and Damasio, A. R. (2000). Long-term sequelae of prefrontal cortex damage acquired in early childhood. Dev. Neuropsychol. 18, 281-296.

Anderson, V., Catroppa, C., Dudgeon, P., Morse, S., Haritou, F., and Rosenfeld, J. (2006). Understanding predictors of functional recovery and outcome thirty-months following early childhood head injury. Neuropsychology 20, 42-57.

Anderson, V., Catroppa, C., Morse, S., Haritou, F., and Rosenfeld, J. (2005). Functional plasticity or vulnerability following early brain injury? Pediatrics 116, 1374-1382.

Anderson, V., Levin, H., and Jacobs, R. (2002). Developmental and acquired lesions of the frontal lobes in children:

might reflect differences in brain pathology rather than age at insult. In order to minimize this risk, we confined our recruitment to children with focal brain pathologies and collected detailed information on brain pathology (e.g., location, laterality), allowing us to control for these potential confounds. We believe that this approach has provided important data to assist in understanding the impact of EBI from an empirical perspective. Of note, we employed a categorical approach to quantifying developmental stage. While these categories reflect central nervous system growth spurts, they are necessarily inexact and may mask specific critical developmental periods. Third, the lack of association between social risk and social outcome is somewhat surprising, and may have been due to choice of social risk measure. Thus, this finding should be interpreted with caution. Finally, while the total sample size provided sufficient power to investigate our primary and secondary aims, it was insufficient to conduct sub-analyses, which may have provided further information about the roles of sub-regions of the brain for social outcome. To extend these findings, prospective, multi-centre research facilitating larger sample sizes is required.

\section{CONCLUSIONS}

This study has explored social outcomes in children with EBI. Our findings demonstrate that these children are at elevated risk of problems in social functioning. While the relationship between development age at insult and outcomes is not as clear as has been identified in studies investigating cognitive outcomes, our results do suggest that children with congenital and perinatal brain insults are most at risk, and children with brain insult during the preschool-age and in late childhood demonstrate relatively good social function. Presence of seizures and family dysfunction was also predictive of aspects of social function following EBI. Finally, although previous research has indicated that lesion characteristics (location, laterality) contribute to cognitive outcome in children, this did not appear to be the case for social outcomes. Surprisingly, social risk also contributed little to social outcome in our sample.

Bohnert, A., Parker, J., and Warschausky, S. (1997). Friendship and social adjustment of children following a traumatic brain injury: an exploratory investigation. Dev. Neuropsychol. $13,477-486$.

Chilosi, A., Pecini, C., Cipriani, F., Brovedani, P., Brizzolara, D., and Ferretti, G. (2005). Atypical language lateralization and early linguistic development in children with focal brain lesions. DMCN 47, 725-730.

Csikszentmihaly, M., and Larson, R. (1984). Being Adolescent. New York, Basic Books.

Demorest, A., Meyer, C., Phelps, E. Gardner, H., and Winner, E. (1984). Words speak louder than actions: Understanding deliberately false remarks. Child Dev. 55, 1527-1534.

Diamond, A., and Taylor, C. (1996). Development of an aspect of executive control: Development of the abilities to remember what I said and to "Do as I say, not as I do". Dev. Psychobiol. 29, 315-334.

Dodge, K., Laird, R., Lochman, J., and Zelli, A. (2002). Multidimensional latent-construct analysis of children's social information processing patterns: correlations, with aggressive problems behavior problems. Psychol. Assess. 14, 60-73.

Eslinger, P., and Biddle, K. (2008). Prefrontal cortex and the maturation of executive functions, cognitive expertise, and social adaptation. In Executive Functions and the Frontal Lobes: A Lifespan Perspective, V. Anderson, R. Jacobs and P. Anderson, eds. (Philadelphia, PA, Taylor \& Francis), pp. 299-316.

Ewing-Cobbs, L., Fletcher, J., Levin, H., Francis, D., Davidson, K., and Miner, M. (1997). Longitudinal neuropsychological outcomes in infants and preschoolers with traumatic brain injury. J. Int. Neuropsychol. Soc. 3, 581-591. 
Ganesalingam, K., Sanson, A., Anderson, V., and Yeates, K. (2006).Self-regulation and social and behavioral functioning following childhood traumatic brain injury. J. Int. Neuropsychol. Soc. 12, 609-621.

Goncu, A., Patt, M., and Kouba, E. (2002). Understanding young children's pretend paln in context. In Blackwell Handbook of Child Social Development, S.C. Hart, ed. (Malden, MA, Blackwell), pp. 418-437.

Goodman, R. (1997). The strengths and difficulties questionnaire: a research note. J. Child Psychol. Psychiatr. 38, 581-586.

Hartel, C., Schilling, S., Sperner, J., and Thyen, U. (2004). The clinical outcomes of neonatal and childhood stroke: review of the literature and implications for future research. Eur. J. Neurol. 11, 431-438.

Howes, C., and Phillipsen, L. (1998). Continuity ion children's relationships with peers. Soc. Dev. 7, 340-349.

Jacobs, R., Harvey, A. S., and Anderson, V. (2007). Executive function following focal frontal lobe lesions: impact of timing of lesion on outcome. Cortex 43, 792-805.

Janusz, J., Kirkwood, M., Yeates, K. O., and Taylor, H. G. (2002). Social problemsolving skills in children with traumatic brain injury: long-term outcomes and prediction of social competence. Child Neuropsychol. 8, 179-194.
Ladd, G., and Pettit, G. (2002). Parenting and the devolopment of children's peer relationships. In Handbook of Parenting, Vol. 5 Practical Issues in Parenting, M. Bornstein, ed. (Mahwah, NJ, Erlbaum), pp. 2269-2309.

Leventer, R., Phelan, E., Coleman, L. Kean, M., Jackson, G., and Harvey, A. (1999). Clinical and imaging features of cortical malfomrations in childhood. Neurology 53, 715-722.

Mellor, D. (2005). Normative data for strengths and difficulties questionnaire in Australia. Aust. Psychol. 40, 215-222.

Miller, I. W., Epstein, N. B., Bishop, D. S., and Keitner, G. I. (1985). The McMaster family assessment device: reliability and validity. J. Marital Fam. Ther. 11, 345-356.

Rholes, W., Ruble, D., and Newman, L. (1990). Children's understanding of self and other: developmental and motivational aspects of perceiving persons in terms of invariant dispositions. In The Handbook of Motivation and Cognition: Foundations and Social Behavior, Vol. II, R. Sorention and E. Higgins, eds (Hillsdale, NJ, LEA), pp. 369-407.

Roberts, G., Howard, K., Spittle, A., Brown, N., Anderson, P., and Doyle, L. (2008). Rates of early intervention serves in very preterm children with developmental disabilities at age
2 years. J. Paediatr. Child Health 44 276-280.

Saxe, R., Carey, S., and Kanwisher, N. (2004). Understanding other minds: linking developmental psychology and functional neuroimaging. Ann. Rev. Psychol. 55, 87-124.

Spencer-Smith, M., and Anderson, V. (2009). Healthy and abnormal development of the prefrontal cortex. Dev. Neurorehabil. 12, 279-297.

Taylor, H. G., Yeates, K., Wade, S., Drotar, D., Stancin, T., and Minich, N. (2002). A prospective study of short- and long-term outcomes after traumatic brain injury in children: behaviour and achievement. Neuropsychology, 16, 15-27.

Van Overwalle, F. (2009). Social cognition and the brain: a meta-analysis. Hum. Brain Mapp. 30, 829-858.

Walker, H., and McConnell, S. (1995). User's Manual for Walker-McConnell Scale of Social Competence and Scholl Adjustment. London, Singular Publishing Group.

Wechsler, D. (1999). Manual for the Wechsler Abbreviated Scale of Intelligence. New York, Psychological Corporation.

Woodward, A. L. (1998). Infants selectively encode the goal object of an actor's reach. Cognition 69, 1-34.

Yeates, K. O., Bigler, E. D., Dennis, M., Gerhardt, C. A., Rubin, K. H., Stancin, T., Taylor, H. G., and Vannatta, K.
(2007). Social outcomes in childhood brain disorder: a heuristic integration of social neuroscience and developmental psychology. Psychol. Bull. 133, 535-556.

Yeates, K. O., Swift, E., Taylor, H. G. Wade, S. L., Drotar, D., Stancin, T., and Minich, N. (2004). Short- and long-term social outcomes following pediatric traumatic brain injury. J. Int. Neuropsychol. Soc. 10, 412-426.

Conflict of Interest Statement: The authors declare that the research was conducted in the absence of any commercial or financial relationships that could be construed as a potential conflict of interest.

Received: 31 August 2009; paper pending published: 13 October 2009; accepted: 23 February 2010; published online: 22 March 2010.

Citation: Greenham M,Spencer-SmithMM, Anderson PJ, Coleman L and Anderson VA (2010) Social functioning in children with brain insult. Front. Hum. Neurosci. 4:22. doi: 10.3389/fnhum.2010.00022

Copyright (c) 2010 Greenham, SpencerSmith, Anderson, Coleman and Anderson. This is an open-access article subject to an exclusive license agreement between the authors and the Frontiers Research Foundation, which permits unrestricted use, distribution, and reproduction in any medium, provided the original authors and source are credited. 